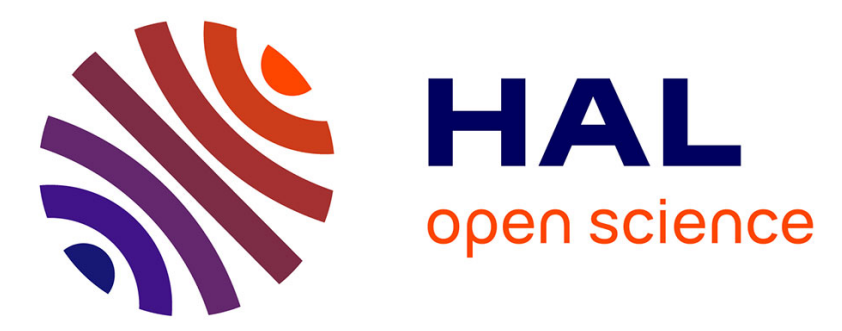

\title{
Comparison of Surrogate Models for Extreme Quantile Estimation in the Context of EMC Risk Analysis
}

T. Houret, Philippe Besnier, S Vauchamp, Philippe Pouliguen

\section{To cite this version:}

T. Houret, Philippe Besnier, S Vauchamp, Philippe Pouliguen. Comparison of Surrogate Models for Extreme Quantile Estimation in the Context of EMC Risk Analysis. Asia-Pacific EMC Symposium, Jun 2019, Sapporo, Japan. hal-02314034

\section{HAL Id: hal-02314034 \\ https://hal.science/hal-02314034}

Submitted on 11 Oct 2019

HAL is a multi-disciplinary open access archive for the deposit and dissemination of scientific research documents, whether they are published or not. The documents may come from teaching and research institutions in France or abroad, or from public or private research centers.
L'archive ouverte pluridisciplinaire HAL, est destinée au dépôt et à la diffusion de documents scientifiques de niveau recherche, publiés ou non, émanant des établissements d'enseignement et de recherche français ou étrangers, des laboratoires publics ou privés. 


\section{Comparison of Surrogate Models for Extreme Quantile Estimation in the Context of EMC Risk Analysis}

\author{
T. Houret ${ }^{1,2}$, P. Besnier ${ }^{1}$ \\ ${ }^{1}$ INSA Rennes, CNRS, IETR \\ UMR 6164, F-35000 \\ Rennes, France
}

\author{
S. Vauchamp ${ }^{2}$ \\ ${ }^{2}$ CEA DAM, \\ F-46500, \\ Gramat, France
}

\author{
P. Pouliguen ${ }^{3}$ \\ ${ }^{3}$ DGA \\ DGA/DS/MRIS, F-75009, \\ Paris, France
}

\begin{abstract}
Various EMC problems may be studied from numerical simulations involving 3D Maxwell equation solvers. However, the EMC risk analysis, either from a susceptibility or emissivity point of view requires various configurations of coupling paths described by important sets of unknown or uncertain parameters. The use of surrogate models is very relevant to speed up the risk analysis process. More specifically, values at risk corresponding to extreme values of relevant fields, currents or voltages are often the most important information with regard to a possible EMC risk. Specific methods such as controlled stratification provide a way to sample the input space of random variables in an efficient way to estimate extreme quantiles of a distribution. However, it requires a simple (i.e. fast calculation time) companion model. This companion model has to be correlated to the reference model in a specific sense. Building a surrogate model would be a possible way. This paper discusses various surrogate models and provides some conclusions about their ability to provide either a direct estimation of extreme quantiles of the response of interest, or a companion model for the controlled stratification method.
\end{abstract}

Keywords-Uncertainty propagation; Monte Carlo; Extreme quantiles; Surrogate models; Controlled stratification

\section{INTRODUCTION}

This communication deals with uncertainty propagation through electromagnetic compatibility models for reliability analysis.

EMC risk analysis, in the context of intentional electromagnetic interference (IEMI), often requires solving Maxwell equations with 3D numerical solvers based on, for instance, method of moments or finite difference time domain. Such models are deterministic and may be considered to provide "exact" solutions, but are very time consuming. At system-level EMC analysis, many inputs are not well known due to epistemic uncertainties. These uncertainties propagate through the model making the output uncertain. The output is therefore described as random variables. The goal is to estimate the distribution of the output for specified input distributions. In the context of IEMI risk analysis, we are particularly interested in output extreme values. Even though extreme values have low likelihood, they mainly contribute to the failure probability.

Monte Carlo (MC) simulation is the standard approach to retrieve the output distribution. A design of experiment (DOE) is set up with many (thousands or more) input realizations (typically from Latin Hypercube Sampling [1]). The model is called for each of them so that the empirical distribution of the output is computed. This approach is simple and very robust but has a very slow convergence especially if extreme values are targeted.

Surrogate models (SMs) are functions that approximate the true model (physical phenomena) and may be calculated from a much more reduced set of realizations (hundreds). Once built, they have a negligible computational cost. Accurate SMs are therefore potential very good alternatives to a standard MC approach.

There are two types of SMs. The first one is considered as physical whereas the second one is probabilistic. To build a physical SM, a good knowledge of the physical phenomena is needed in order to make assumptions and approximations. Such SMs are therefore problem specific. The second type of SM does not require any physical knowledge and is therefore virtually universal. However, many SMs exist (including many variants) and their relative performances must be assessed.

The output distribution can be estimated by propagating the uncertainty through the SM as a substitute of the reference model. SMs may also be used in addition to other techniques targeting the output distribution tail, such as controlled stratification (CS) [2], subset simulation [3], importance sampling [4]. The CS has been successfully applied to an EMC case study in [5] but the SM was specific and based on physical knowledge. In fact, CS performances rely on the strong correlation between the SM and the reference model. In such conditions, an input producing an extreme event with the SM is likely to produce also an extreme event of the reference model.

In this study we chose five of the most popular SMs: Kriging (KRI) [6], Polynomial Chaos Expansion (PCE) [7], Support Vector Machine (SVM) [8], Neural Network (NN) [9] and Polynomial Chaos Expansion with Kriging (PCE-KRI) [10]. These SMs have recently been used in an EMC context. In [11], SVM is compared to PCE to estimate the output distribution. In [12] a sparse PCE in a high dimension problem is compared to MC to estimate the output distribution. In [13] an adaptive kriging is compared to classical reliability methods (FORM, Importance Sampling, Subset Simulation) to estimate the output distribution tail.

To our knowledge, no SMs performance comparison in terms of error and correlation has been made in the particular case of estimating extreme values in EMC applications. In this paper we aim at undertaking a fair comparison of the SMs with EMC inspired analytical models. Our purpose is to identify the best SM for CS. This paper also draws some conclusions about the legitimacy of using the CS rather than a stand-alone SM. 
First we specify which SMs variant are used, their associated parameters, and the performance measurement. Appropriate references are added for theoretical details. Then, we present a performance comparison of the SMs on two application cases. Finally, a conclusion and perspectives are given.

\section{IMPLEMENTATION DETAILS}

\section{A. Support Vector Machine (SVM) [8]}

We used the Matlab UQlab SVM implementation [14] with the matern-5_2 kernel, the smooth leave-one-out estimation method and the Broyden-Fletcher-Goldfarb-Shanno (BFGS) optimization algorithm (200 iterations maximum) to select the penalty term, insensitive tube and kernel parameters.

\section{B. Polynomial Chaos Expansion (PCE) [7]}

We used the Matlab UQLab implementation [15] with a least angle regression computation strategy and the adaptive maximum degree (from 1 to 3 ). In addition to select the best maximum degree, this algorithm discards polynomials with negligible impact on the output.

\section{Kriging (KRI) [6]}

We used the Matlab UQlab kriging implementation [16] with ordinary trend, a matern-5_2 correlation function, the maximum likelihood estimation method and the limited memory variant of BFGS optimization algorithm (maximum of 200 iterations).

\section{Polynomial Chaos Expansion-Kriging (PCE-KRI) [10]}

The PCE-KRI SM is a hybridization between the two previously presented SMs: KRI and PCE. The PCE-KRI is in fact a kriging in which the trend is a PCE. The benefits of both SMs (local error and Sobol's indices) are therefore reunited. We used the Matlab UQlab implementation [17] with the same characteristic used for KRI and PCE alone.

\section{E. Neural Network (NN) [9]}

We used the Matlab Neural Net fitting Toolbox. The search for the optimal weights/bias values was made with the Levenberg-Marquardt (LM) algorithm [18] with a Bayesian regularization [19]. The data is split into a train set $(80 \%)$, a validation set $(10 \%)$ and a test set $(10 \%)$. The LM parameters are: $\mu_{0}=100, \mu_{\mathrm{inc}}=5, \mu_{\mathrm{dec}}=0.5$. The maximum of validation failures is set to 100 and the maximum time spent is limited to 15 seconds. We chose arbitrary two hidden layers. The best number of neurons (resulting the lowest test error) in each layer is found with a grid search from 10 to 40 (step of 10) for the first layer and from 5 to 35 (step of 10) in the second layer.

\section{F. Performance measurement}

Performances of each SM are evaluated thanks to both the relative generalized error in the distribution tail (Err) and the statistical order correlation with the initial model $(\rho)$. As long as the correlation between the SM and the model is high enough, the CS can be very effective in estimating extreme values. In fact, the SM error does not systematically affect the CS performances. If the error is low enough the SM could be used directly to estimate extreme quantiles (uncertainty propagation through the SM). It might not be interesting to use the CS in addition to the SM in that case. On the contrary, if the error is large but the correlation remains good, the CS might be better than the SM alone.

A SM is built with a small DOE sample of size $n$. An independent, much larger test sample of size $N(10000)$ is then used to evaluate the SMs performances. Among those $N$ realizations, Next ones belong to the distribution tail. The lower tail gathers the set of output realizations yext ${ }_{i}$ smaller than $y_{\alpha}$ (the model $\alpha$ quantile). In that case, we arbitrary set $\alpha$ in the range $1-10 \%$. The upper tail gathers realizations greater than $y_{\alpha}$. In that case, $\alpha$ is arbitrary set in the range $90-99 \%$. The generalized error at extreme values is the relative root mean square error of the difference between predicted SM extreme values $\hat{y} \mathrm{ext}_{i}$ and the true extreme values yext ${ }_{i}$ :

$$
E r r=\sqrt{\frac{1}{N e x t} \sum_{i=1}^{N e x t}\left(\left(\frac{\hat{y} \operatorname{ext}_{i}-\text { yext }_{i}}{\text { yext }_{i}}\right)^{2}\right)}
$$

The correlation between the model $(Y)$ and the SM $(Z)$ in the CS formulation is computed with all the $N$ values at given probabilities $\alpha$. The values $y_{\alpha}$ and $z_{\alpha}$ are the $\alpha$ quantiles of the model and the SM, respectively. The correlation is given by:

$$
\rho(\alpha)=\frac{\mathrm{P}\left(Y \leq y_{\alpha} \mid Z \leq z_{\alpha}\right) \alpha-\alpha^{2}}{\alpha-\alpha^{2}}
$$

A quick interpretation of (2) follows. If $Y$ and $Z$ are independent, the conditional probability in (2) becomes the product of marginal probabilities and the correlation is null: $P\left(Y \leq y_{\alpha} \mid Z \leq z_{\alpha}\right) \rightarrow P\left(Y \leq y_{\alpha}\right) P\left(Z \leq z_{\alpha}\right)=\alpha^{2} \Leftrightarrow \rho \rightarrow 0$. Another situation is that of a negative correlation: each extreme value of $Z$ would not correspond to an extreme value of $Y$. Therefore: $\quad P\left(Y \leq y_{\alpha} \mid Z \leq z_{\alpha}\right) \rightarrow 0 \Leftrightarrow \rho(\alpha) \rightarrow \frac{-\alpha^{2}}{\alpha-\alpha^{2}}<0$. Finally, in case of perfect correlation, every extreme value of $Z$ would correspond to an extreme value of $\mathrm{Y}$, which means: $\left(Y \leq y_{\alpha} \mid Z \leq z_{\alpha}\right) \rightarrow 1 \Leftrightarrow \rho \rightarrow 1$. As a result, we look for situation where $\rho(\alpha)$ is high enough.

These performances indicators depend on the sampled DOE. Therefore, the distribution of $\operatorname{Err}$ and $\rho(\alpha)$ is estimated from 100 DOEs of size $n$.

\section{SM PERFORMANCES COMPARISON}

\section{A. PCB trace field emission}

\section{1) Model Inputs/Output}

The model is the electrical far-field magnitude radiated by a PCB trace, loaded at each end, above an infinite ground plane. See Fig. 1 in [20].

The 11 random variable inputs are: the frequency $(f)$, geometrical characteristics (the substrate high $(h)$, the trace width/length $W / l$ ), electrical characteristics (substrate permittivity $\varepsilon_{r}$, voltage source $V_{s}$, impedance source $Z_{s}$, impedance load $Z_{l}$ ), and the position where the field is measured (spherical coordinates $r, \theta$, and $\varphi$ ). Each input follows a Gaussian distribution centered at their nominal value with a relative standard deviation of $10 \%$. The nominal values are chosen so that the trace appears as a quarter wavelength 
transmission line: $f=404 \mathrm{MHz}, \quad h=0.775 \mathrm{~cm}, \quad W=0.51 \mathrm{~cm}$, $l=10.16 \mathrm{~cm}, \varepsilon_{r}=4.6, \mathrm{Vs}=1 \mathrm{~V}, Z_{s}=50 \Omega, Z_{l}=1 \Omega, r=3 \mathrm{~m}, \varphi=\theta=2 \pi$.

The model output is the radiated field magnitude computed from (23) in [20]. The output is plotted as a function of frequency in Fig. 1 where red circles indicate output realizations above the $y_{90 \%}$ quantile.

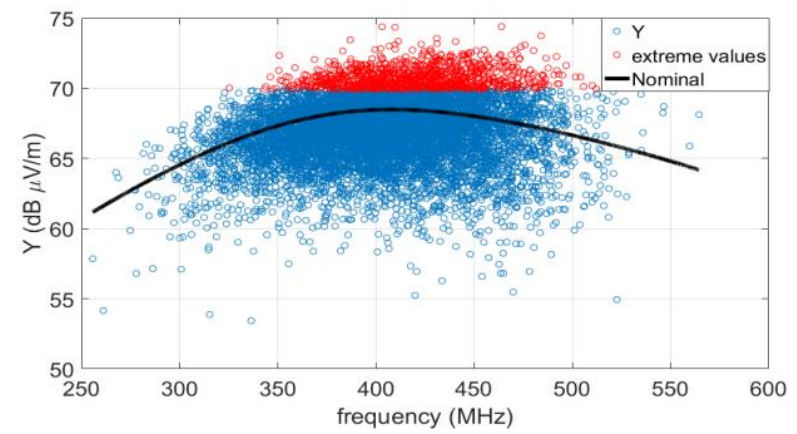

Fig. 1. PCB radiated field for 10000 random inputs (red points for $Y>y_{90 \%}$ ). Field variation with frequency while other inputs are set to their nominal value.

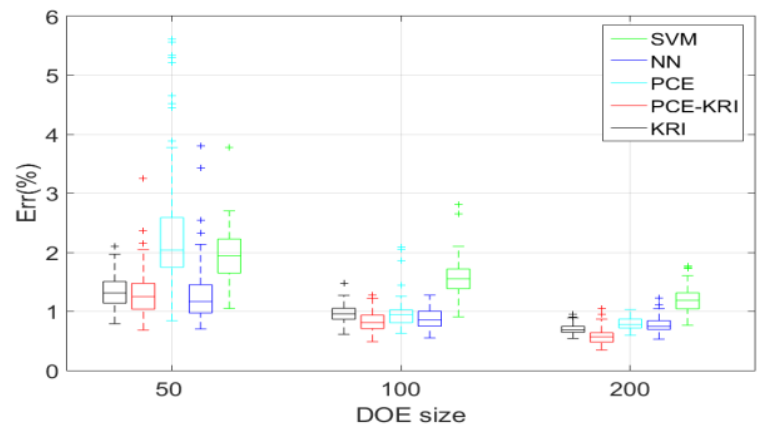

Fig. 2. PCB radiation: generalized errors distributions for extreme values $\left(Y>y_{90 \%}\right)$ in function of DOE sizes $(n)$.

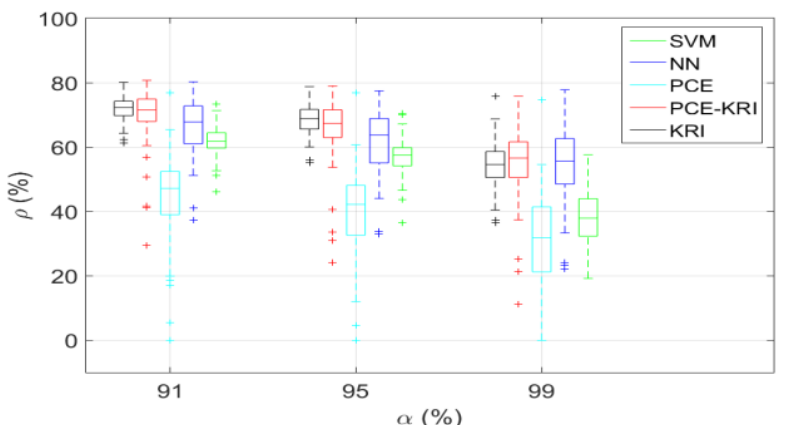

Fig. 3. PCB radiation: correlations distributions for a DOE of size $n=50$ in function of the probability $\alpha$.

\section{1) SM performances}

In Fig. 2, the SM errors are already low even for small $n$. As $n$ increases, every SM errors decreases and are less spread. The PCE_KRI has the lowest error, followed by the KRI. The SVM is better than PCE and NN for small $n$ but becomes the worst at larger $\mathrm{n}$. Therefore the ranking from the lowest error to the greatest is: PCE_KRI, KRI, SVM, NN, PCE for small $n$ (50 and 100) and for larger $n$ (200) SVM falls to last place.

The correlation for small $n$ (50), computed at the $\alpha$ probability, is plotted in Fig. 3. As $\alpha$ increases, every SM correlation decreases and is more spread as expected. The
PCE_KRI and KRI correlation are the highest. The PCE has the lowest correlation. The ranking from the highest correlation to the lowest is the same as the ranking for the error (at small $n)$.

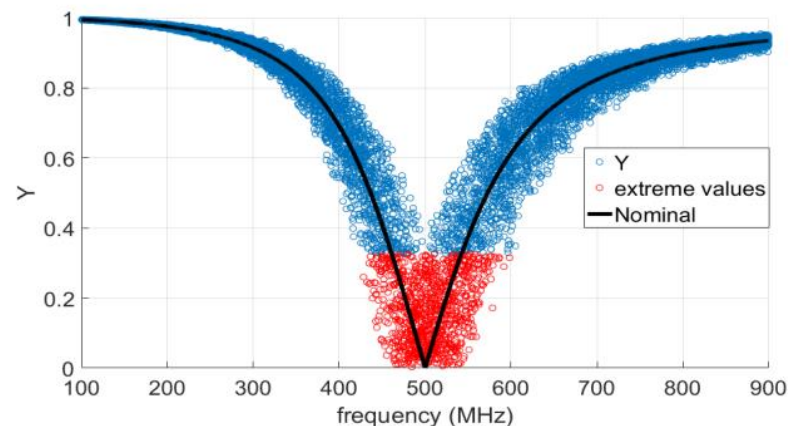

Fig. 4. Reflection coefficient (in linear) for 10000 random inputs (red points for $Y \leq y_{10 \%}$ ). Reflection variation with frequency while other inputs are set to their nominal value.

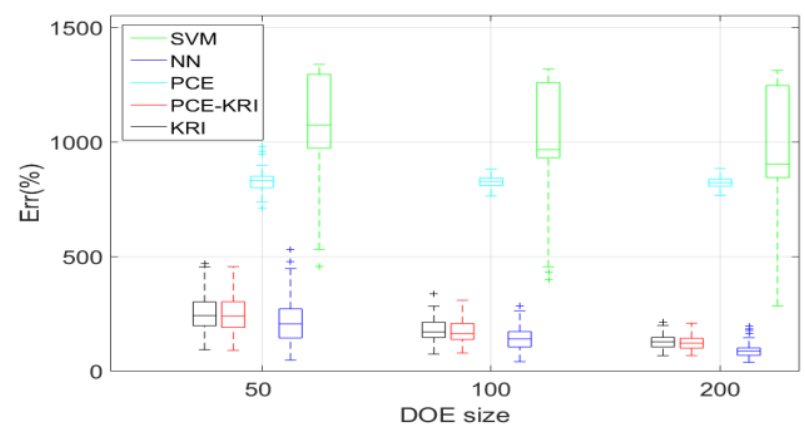

Fig. 5. RLC reflection: generalized errors distributions for extreme values $\left(Y \leq_{10 \%}\right)$ in function of DOE sizes $(n)$.

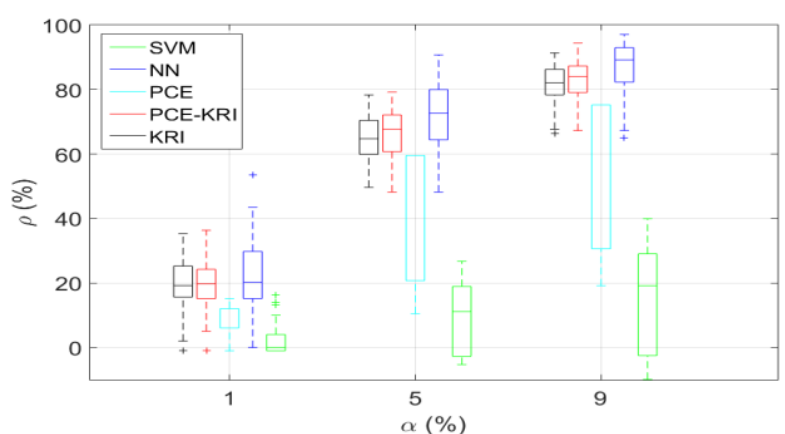

Fig. 6. RLC reflection: correlations distributions for a DOE of size $n=50$ in function of the probability $\alpha$.

Although the ranking according to the error is the same as the ranking according to the correlation, the error is already very low $(\leq 2 \%)$ whereas the correlation is not very high. We suspect that in the PCB case, the CS would not be needed as the PCE_KRI already approximates well the model in the distribution tail. In fact, due to moderate correlation, the CS with the PCE_KRI might be worse than the PCE_KRI alone.

\section{B. Reflection of a RLC serial circuit}

\section{1) Model Inputs/Output}

The second studied model is the reflection coefficient of a RLC serial circuit. The 4 random inputs are: the frequency, the resistance, the capacitance and the inductance. The load impedance is fixed $\left(Z_{l}=50 \Omega\right)$ as well as the input voltage $(1 \mathrm{~V})$. 
The frequency is uniformly distributed from 100 to 900 $\mathrm{MHz}$. The resistance, capacitance and inductance are uniformly distributed between $90 \%$ and $110 \%$ of their nominal values. The model output (the reflection coefficient) is plotted in Fig. 4. The nominal values $(\mathrm{R}=50 \Omega, \mathrm{C}=1.5 \mathrm{e}-12 \mathrm{~F}$ and $\mathrm{L}=6.75 \mathrm{e}-8 \mathrm{H})$ are chosen to reach a resonance at $500 \mathrm{MHz}$.

TABLE I. RLC REFLECTION CASE: PCE-KRI MEAN CORRELATION (\%) AND MEAN ERROR (\%)

\begin{tabular}{|c|c|c|c|}
\hline $\boldsymbol{n}$ & $\mathbf{5 0}$ & $\mathbf{1 0 0}$ & $\mathbf{2 0 0}$ \\
\hline$\rho$ & 58.9 & 69.9 & 77.0 \\
\hline$E r r$ & 243.3 & 169.9 & 121.9 \\
\hline
\end{tabular}

2) SM performances

In Fig. 5, every SM has a very high error. SVM and PCE yield to the most important errors. KRI, PCE_KRI and NN are similar although NN performs slightly better. The ranking according to the error is: (PCE_KRI, KRI, NN), PCE, SVM. "KRI is almost identical to PCE_KRI, which is a KRI with a PCE trend. This indicates that the random process has either a constant trend or that the PCE cannot estimate it. From a study not reported here for brevity, we found out that the random process trend can be indeed considered constant."

In Fig. 6, the ranking of the correlation is the same as for the error. Negative correlations encountered with the SVM, are not impossible but obviously not desired.

In Table I, the mean error at extreme values $\left(Y \leq y_{10 \%}\right)$ is reported with the mean correlation for extreme probabilities $(\alpha \leq 10 \%)$. The errors are very high whereas the correlations are not very low. In that case, we suspect that the CS might be useful.

\section{CONCLUSION}

In this paper we investigated four SMs, candidates for the $\mathrm{CS}$ approach in order to estimate extreme quantiles when brute force $\mathrm{MC}$ is too expensive.

In most cases the PCE_KRI performed the best, closely followed or similarly as the KRI. Like KRI, PCE_KRI provides a local error estimation of its prediction. An adaptive refinement of the DOE could be easily implemented and therefore would most likely improve the performances. Moreover the PCE-KRI has also sensitivity indices, unlike KRI. In case of very high dimensions, the sparse PCE variant [21] and the Partial Least Square in Kriging [22] could be used. Even though the NN is appealing, it does not provide a local error or sensitivity analysis.

We found out in the RLC application example that with the best SM (PCE_KRI) the generalized error in the output distribution tail is very important (above 120\%), the correlation is in fact not that low (above 58\%). This encourages the use of the CS rather the SM alone. In the PCB radiation case example, the error is already low $(\leq 2 \%)$ with the best SM (PCE_KRI) whereas the correlation is not very high. In that case, the CS may be less interesting than the SM alone.

As a conclusion, we retain the PCE-KRI to supply the control stratification method CS. Test will be undertaken in the near future.
The authors would like to thank the support from the DGA.

\section{REFERENCES}

[1] M. Stein, "Large Sample Properties of Simulations Using Latin Hypercube Sampling," Technometrics, vol. 29, no. 2, p. 143, May 1987.

[2] C. Cannamela, J. Garnier, and B. Iooss, "Controlled stratification for quantile estimation,” Ann. Appl. Stat., vol. 2, no. 4, pp. 1554-1580, Dec. 2008.

[3] X. Huang, J. Chen, and H. Zhu, "Assessing small failure probabilities by AK-SS: An active learning method combining Kriging and Subset Simulation," Struct. Saf., vol. 59, pp. 86-95, Mar. 2016.

[4] B. Echard, N. Gayton, M. Lemaire, and N. Relun, "A combined Importance Sampling and Kriging reliability method for small failure probabilities with time-demanding numerical models," Reliab. Eng. Syst. Saf., vol. 111, pp. 232-240, Mar. 2013.

[5] M. Larbi, P. Besnier, and B. Pecqueux, "The Adaptive Controlled Stratification Method Applied to the Determination of Extreme Interference Levels in EMC Modeling With Uncertain Input Variables," IEEE Trans. Electromagn. Compat., vol. 58, no. 2, pp. 543-552, Apr. 2016.

[6] C. E. Rasmussen and C. K. I. Williams, Gaussian processes for machine learning, 3. print. Cambridge, Mass.: MIT Press, 2008.

[7] T. Crestaux, O. Le Ma1 tre, and J.-M. Martinez, "Polynomial chaos expansion for sensitivity analysis," Reliab. Eng. Syst. Saf., vol. 94, no. 7, pp. 1161-1172, Jul. 2009.

[8] A. J. Smola and B. Schölkopf, "A tutorial on support vector regression,” Stat. Comput., vol. 14, no. 3, pp. 199-222, Aug. 2004.

[9] M. Nielsen, Neural Network and Deep Learning. 2018.

[10] R. Schobi, B. Sudret, and J. Wiart, "POLYNOMIAL-CHAOSBASED KRIGING," Int. J. Uncertain. Quantif., vol. 5, no. 2, pp. 171-193, 2015.

[11] R. Trinchero, P. Manfredi, I. S. Stievano, and F. G. Canavero, "Machine Learning for the Performance Assessment of High-Speed Links," IEEE Trans. Electromagn. Compat., vol. 60, no. 6, pp. 1627 1634, Dec. 2018.

[12] M. Larbi, I. S. Stievano, F. G. Canavero, and P. Besnier, "Variability Impact of Many Design Parameters: The Case of a Realistic Electronic Link," IEEE Trans. Electromagn. Compat., vol. 60, no. 1, pp. 34-41, Feb. 2018.

[13] T. Bdour, C. Guiffaut, and A. Reineix, "Use of Adaptive Kriging Metamodeling in Reliability Analysis of Radiated Susceptibility in Coaxial Shielded Cables," IEEE Trans. Electromagn. Compat., vol. 58, no. 1, pp. 95-102, Feb. 2016.

[14] R. Schöbi, S. Marelli, and B. Sudret, "UQLab user manual - Support vector machines for regression.” 2017.

[15] S. Marelli and B. Sudret, "UQLab user manual - Polynomial Chaos Expansions." 2017.

[16] R. Schöbi, S. Marelli, and B. Sudret, "UQLab user manual - Kriging (Gaussian process modelling)." 2017.

[17] R. Schöbi, S. Marelli, and B. Sudret, "UQLab user manual - PCKriging." 2017.

[18] M. T. Hagan and M. B. Menhaj, "Training feedforward networks with the Marquardt algorithm," IEEE Trans. Neural Netw., vol. 5, no. 6, pp. 989-993, Nov. 1994.

[19] F. Dan Foresee and M. T. Hagan, "Gauss-Newton approximation to Bayesian learning," in Proceedings of International Conference on Neural Networks (ICNN'97), Houston, TX, USA, 1997, vol. 3, pp. 1930-1935.

[20] M. Leone, "Closed-Form Expressions for the Electromagnetic Radiation of Microstrip Signal Traces," IEEE Trans. Electromagn. Compat., vol. 49, no. 2, pp. 322-328, May 2007.

[21] G. Blatman and B. Sudret, "An adaptive algorithm to build up sparse polynomial chaos expansions for stochastic finite element analysis," Probabilistic Eng. Mech., vol. 25, no. 2, pp. 183-197, Apr. 2010.

[22] M. A. Bouhlel, N. Bartoli, A. Otsmane, and J. Morlier, "Improving kriging surrogates of high-dimensional design models by Partial Least Squares dimension reduction," Struct. Multidiscip. Optim., vol. 53, no. 5, pp. 935-952, May 2016. 